\title{
The effect of cognitive status and visuospatial performance on affective theory of mind in Parkinson's disease
}

This article was published in the following Dove Press journal:

Neuropsychiatric Disease and Treatment

5 August 2013

Number of times this article has been viewed

\section{Audrey McKinlay ${ }^{1,2}$ \\ Michelle Albicini ${ }^{2}$ \\ Phillip S Kavanagh ${ }^{3}$}

'Department of Psychology, University of Canterbury, Christchurch, New Zealand; ${ }^{2}$ Department of Psychology and Psychiatry, Monash University, Clayton, VIC, Australia; ${ }^{3}$ School of Psychology, Social Work and Social Policy, University of South Australia, Adelaide, SA, Australia
Correspondence: Audrey McKinlay School of Psychology and Psychiatry Monash University, Building 17,

Clayton Campus, Wellington Road, Monash University, Clayton,

VIC 3800, Australia

Tel +6I 399053945

Email audrey.mckinlay@monash.edu
Abstract: It is now well accepted that theory of mind (ToM) functioning is impaired in Parkinson's disease (PD) patients. However, what remain unknown are the functions that underlie this impairment. It has been suggested that cognitive skills may be key in this area of functioning; however, many of the cognitive tests used to assess this have relied on intact visuospatial abilities. This study aimed to examine whether deficits in ToM were generated by cognitive or visuospatial dysfunction and the mediating effect of visuospatial function on ToM performance. Fifty PD patients ( 31 male, 19 female; mean age $=66.34$ years) and 49 healthy controls ( 16 male, 33 female; mean age $=67.29$ years) completed a ToM task (reading the mind in the eyes) and visuospatial task (line orientation). The results revealed that current cognitive status was a significant predictor for performance on the ToM task, and that $54 \%$ of the total effect of cognitive status on ToM was mediated by visuospatial abilities. It was concluded that visuospatial functioning plays an important mediating role for the relationship between executive dysfunction and affective ToM deficits in PD patients, and that visuospatial deficits may directly contribute to the presence of affective ToM difficulties seen in individuals with PD.

Keywords: Parkinson's disease, theory of mind, visuospatial function, social cognition, executive function

\section{Introduction}

Parkinson's disease (PD) is a neurodegenerative disorder marked by motor symptoms including rigidity, bradykinesia, postural problems, and resting tremor. ${ }^{1}$ Nonmotor symptoms are also a frequent feature, including executive dysfunction, cognitive decline, and visuospatial deficits, which can be equally disabling for individuals with PD. ${ }^{1,2}$ Deficits in theory of mind (ToM), the ability to attribute mental states to one's self and others, are also common. Indeed, the cognitive deficits exhibited in PD are said to resemble those produced after frontal lobe damage. ${ }^{3}$ Although not well understood, deficits in ToM are important, as difficulties in this area may reduce the individual's sensitivity to social cues, leading to social isolation and impacting on quality of life. ${ }^{4,5}$ However, it remains unclear whether ToM deficits in PD are due to cognitive decline associated with the disorder, ${ }^{6}$ or visuospatial deficits which also appear early in the disease process.

As mentioned above, a series of studies have highlighted that ToM is impaired in some patients with PD. For instance, participants with PD have been reported to perform more poorly on first-order and second-order belief ToM tasks compared to healthy controls. ${ }^{4-6}$ Furthermore, similar studies have demonstrated worse performance by those with PD compared to individuals without PD on ToM tasks involving cartoons, ${ }^{7}$ 
attributing emotions, ${ }^{8}$ recognizing facial expressions, ${ }^{9}$ and the "reading the mind in the eyes" task. ${ }^{10}$ In an interesting study, irony comprehension and ToM processing were examined in individuals with PD. ${ }^{11}$ Pragmatic stories involving either irony or lying were presented to participants with PD and healthy controls, and the results indicated that PD participants demonstrated deficits in the ability to distinguish between lying versus ironic intent. Further, the PD patients were also impaired when answering questions regarding second-order beliefs of the story characters. ${ }^{11}$

While studies have highlighted an overall deficit of ToM in PD patients, others have attempted to differentiate the subcomponents of ToM. As such, research has identified two subcomponents: cognitive ToM, the ability to understand others' intentions and beliefs, and affective ToM, the ability to identify the emotional states of others. ${ }^{12}$ Further, studies have reported these subcomponents to be related to different brain regions, with cognitive ToM thought to be mediated by the dorsolateral frontostriatal circuitry, and affective ToM primarily by the orbital frontostriatal circuitry. ${ }^{8}$

Through the use of different ToM tasks, studies have compared performance of PD and non-PD participants in cognitive and affective ToM functioning, and while authors have reported only deficits in cognitive ToM, ${ }^{13}$ others have also indicated additional decreased performance on affective ToM functioning. ${ }^{8,12}$ Bodden et al ${ }^{12}$ specifically investigated affective and cognitive ToM functioning in $\mathrm{PD}$, finding that participants with PD scored significantly lower on both the cognitive and affective ToM subscales but not on the control (memory and facts) items.

In light of the above findings, there is now a focus on identifying the potential correlates of ToM deficits in patients with $\mathrm{PD}$, and it has been suggested that problems with ToM reflect the presence of cognitive deficits. ${ }^{6}$ In a study investigating decision-making in patients with PD, performance on tasks involving ToM, executive functioning, and decision-making revealed that while participants with PD exhibited worse performance than controls on all three outcomes, ToM and decision-making did not correlate with executive functioning. ${ }^{14}$ Considering the large reliance on visual-processing in the measures of ToM, such as recognition of emotional expressions, facial processing, and the reading the mind in the eyes task, it may be suggested that visuospatial functioning deficits mediate the relationship between ToM deficits and PD. The aim of this study was to explicitly examine whether deficits in ToM were generated by cognitive or visuospatial dysfunction and the mediating effect of visuospatial function on performance on a ToM task.

\section{Methods}

\section{Participants and procedure}

Ninety-nine participants were recruited from a database of PD patients ( 31 males, 19 females; mean $[\mathrm{M}]$ age $=66.34$ years, standard deviation $[\mathrm{SD}]=6.71$ years) and healthy controls ( 16 males, 33 females; $M$ age $=67.29$ years, $\mathrm{SD}=5.73$ years $)$ from the greater Canterbury region. A neurologist who specialized in movement disorders diagnosed the $50 \mathrm{PD}$ patients. Inclusion criteria: (a) a diagnosis of idiopathic Parkinson's disease, assessed at the Hoehn and Yahr ${ }^{15}$ stage $1-1 \mathrm{~V}$; (b) between 50 and 80 years of age; (c) adequate or corrected hearing and vision (self-report checked by examiner); (d) no signs of overt dementia, with mental status examine score of $>25 .{ }^{16}$ Exclusion criteria comprised a history of: (a) moderate/severe traumatic brain injury; (b) stroke or other neurological impairments other than PD; (c) major medical illness; (d) alcohol or substance abuse; (e) currently taking medications known to have a significant effect on the central nervous system (other than medications prescribed for the control of PD symptoms). All PD patients were on optimal levels of anti-Parkinsonian medication during participation.

The study was approved by the local ethics committee, and all subjects gave written consent prior to participation. Testing was conducted at the University of Canterbury.

\section{Measures}

- Theory of mind task - reading the mind in the eyes task. ${ }^{17}$ Participants were presented with a partial picture of a face showing only the eye-region, and at each of the four corners of the picture was a word. They were then asked to make a judgment about which one of the words most closely matched what the person in the picture might be thinking or feeling. The task consists of 36 test items which were scored 0 for incorrect and 1 for correct choices.

- Visuospatial ability, assessed using the judgment of line orientation test. ${ }^{18}$ This test is frequently used to assess visuospatial function, whereby participants are presented with a booklet containing a series of card pairs. One of the card pairs, the response card, was displayed at the bottom of the page and contained an array of eleven numbered lines (3.8 cm in length), and each was separated by an angle of 18 degrees. The participant was asked to identify the orientation of two lines on the bottom card by identifying the two lines with the same orientation from the eleven-line array. Each participant was presented with five practice items and 30 tests items. Responses for each line 
pair were scored 0 for an incorrect response and 1 for a correct response.

- Symptoms of depression, assessed with the Beck Depression Inventory, 2nd edition (BDI-II). ${ }^{19}$ The BDI-II is a brief, 21 item, self-report questionnaire that assesses an individual's mood over the previous 2-week period. Each item was rated on a four-point scale from 0-3. Higher scores indicated the presence of a greater number of depressive symptoms.

- Cognitive status, assessed using the modified mini mental state exam. ${ }^{16}$ This test involves 30 questions and examines cognitive aspects of mental functioning such as orientation, memory, attention, and ability to follow complex commands. Scores higher than or equal to 25 indicate normal cognitive functioning.

See Table 1 for clinical information broken down by demographic characteristic for each group. Information has been provided separately for males and females.

\section{Results \\ Preliminary analyses}

To determine if there were differences between PD patients and controls on key variables (age, depression, current cognitive status), a $2 \times 2$ (sex $\times$ disease interaction [PD versus control]) multivariate analysis of variance was conducted with age, BDI-II scores, and modified mini mental state scores as the dependent variables. As expected, the results revealed a significant main effect for disease on current cognitive status with PD patients $(\mathrm{M}=94.31)$ demonstrating a significantly lower level of cognitive functioning than healthy controls $(\mathrm{M}=96.98)\left(F[1,95]=9.21, P=0.003, \eta_{\mathrm{p}}{ }^{2}=0.09\right)$. There was also a significant main effect for disease on symptoms of depression with PD patients $(M=8.63)$ endorsing greater levels of depression symptomatology than healthy controls $(\mathrm{M}=4.47)\left(F[1,95]=13.42, P=0.001, \eta_{\mathrm{p}}{ }^{2}=0.13\right)$. Finally, the results revealed a significant sex $\times$ disease interaction for age, with male controls $(\mathrm{M}=69.50$ years $)$ older than male PD patients $(\mathrm{M}=65.39$ years $)$, whereas female controls

Table I Clinical profile of participants by demographics

\begin{tabular}{llll}
\hline & 3MS & H and $\mathbf{~}$ & BDI-II \\
& M (SD) & M (SD) & M (SD) \\
\hline PD males & $94.52(5.00)$ & $2.26(0.82)$ & $8.52(6.42)$ \\
PD females & $94.11(4.48)$ & $2.08(0.84)$ & $8.74(7.13)$ \\
Control males & $96.69(3.84)$ & - & $4.81(4.31)$ \\
Control females & $97.27(3.18)$ & - & $4.12(3.20)$ \\
\hline
\end{tabular}

Abbreviations: 3MS, modified mini mental state examination; BDI-II, Beck Depression Inventory, 2nd edition; $\mathrm{H}$ and $\mathrm{Y}$, Hoehn and Yahr stage; M, mean; $\mathrm{SD}$, standard deviation; PD, Parkinson's diease.
$(\mathrm{M}=66.21$ years) were younger than female $\mathrm{PD}$ patients $(\mathrm{M}=67.90$ years $)\left(F[1,95]=5.00, P=0.028, \eta_{\mathrm{p}}{ }^{2}=0.05\right)$. Post hoc analyses did not reveal significant differences between these means. Given the significant differences in levels of depression symptomatology for PD versus controls, the significant $\operatorname{sex} \times$ disease interaction for age, and the unequal distribution of males and females across the groups, BDI-II scores and sex were entered as covariates for all the main analyses.

\section{Effect of cognitive functioning on theory of mind}

To test the prediction that current cognitive status is predictive of ToM, independent of PD presence, ToM was regressed on cognitive status and disease, while controlling for sex and depression symptomology. The results revealed, as expected, current cognitive status was a significant predictor of ToM ( $\beta=0.31, t=3.11, P=0.003)$, with lower cognitive functioning indicative of ToM deficits. Further, disease was also an independent predictor of ToM $(\beta=0.233, t=2.14$, $P=0.035)$, with PD patients having significantly lower levels of ToM than controls.

\section{Mediational analyses}

To test the prediction that the effect of cognitive functioning on ToM is mediated by deficits in visuospatial skills for PD patients, a bootstrap serial mediational analysis was conducted following the procedures from Hayes. ${ }^{20}$ ToM was entered as the dependent variable, disease as the independent variable, current cognitive status and visuospatial abilities as the mediator, and depression symptomology and sex as covariates. The results (shown in Figure 1), revealed the total effect of disease on ToM $(b=3.61, P=0.003)$ became nonsignificant with the introduction of cognitive functioning and visuospatial abilities into the model (total direct effect: $b=-1.28, P=0.27)$.

The total indirect effect of disease on ToM through cognitive function and visuospatial abilities was significant, with a point estimate of $-2.33,95 \%$ confidence interval (CI) -3.86 to -0.99 . The specific indirect effect of disease on ToM through both cognitive functioning and visuospatial abilities was significant (effect estimate: $-0.77,95 \% \mathrm{CI}-1.67$ to -0.20). The specific indirect effect of disease on visuospatial abilities through cognitive functioning was not significant (effect estimate: $-0.30,95 \% \mathrm{CI}-1.67$ to 0.69 ). The specific indirect effect of cognitive functioning on ToM through visuospatial abilities was significant (effect estimate: -1.25 , $95 \% \mathrm{CI}-2.54$ to -0.24$)$. These results indicate that although 


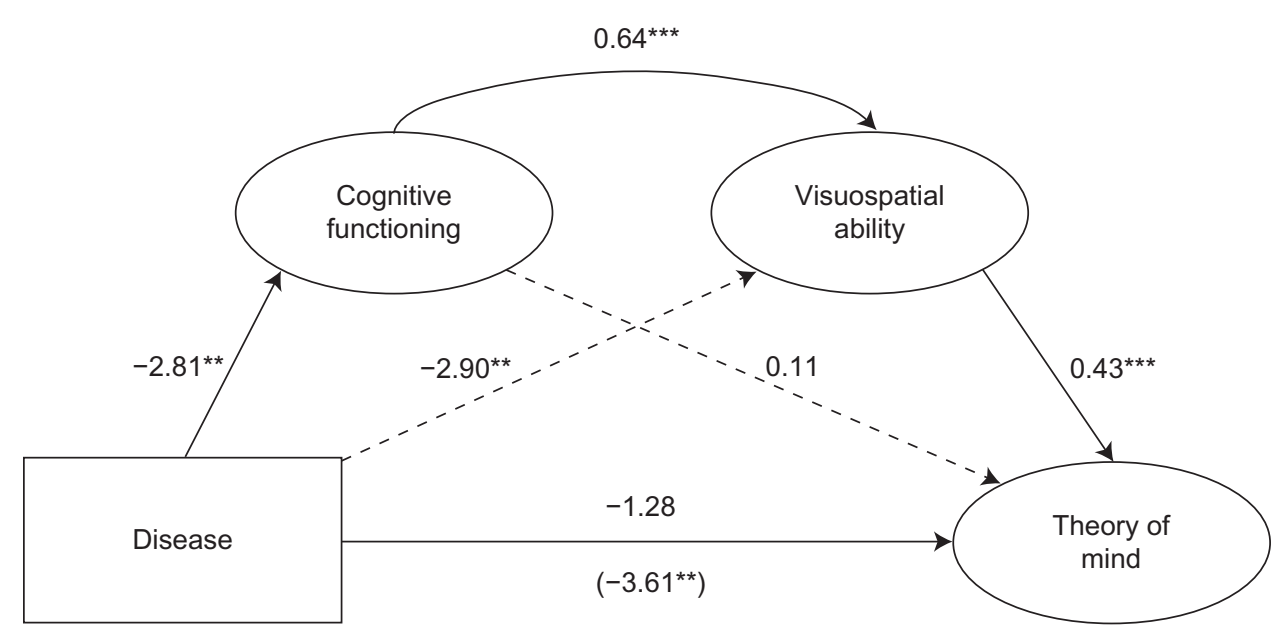

Figure I Current cognitive functioning and visuospatial abilities jointly mediate the effects of Parkinson's disease on affective theory of mind, controlling for sex and depression symptomology.

Notes: All numbers are unstandardized coefficients. The path in parentheses indicates the direct association between disease and theory of mind. ${ }^{* *} P<0.0 \mathrm{I} ;{ }^{* * * k} P<0.00 \mathrm{I}$.

visuospatial ability mediates the effects of cognitive functioning on ToM, more importantly both cognitive functioning and visuospatial ability work in conjunction to mediate the effects of disease on ToM, with the path from disease to visuospatial ability not mediated by cognitive functioning. In sum, PD is associated with lower levels of cognitive functioning, which in turn effects visuospatial functioning, which in turn influences ToM. The direct effects of disease on ToM and cognitive functioning on ToM are all accounted for by visuospatial ability.

\section{Discussion}

The aim of this study was to examine whether ToM deficits detected in patients with PD were associated with cognitive status or visuospatial dysfunction, and to explore the mediating effect of visuospatial function on performance with a ToM task. We found that while current cognitive status was a significant predictor for performance on the ToM task, the association between PD and ToM and the total effect of cognitive status on ToM was being mediated by visuospatial abilities.

Our research is consistent with a number of other studies. $^{8-10,12}$ However, there is considerable controversy regarding the timing of different ToM deficits, with some arguing that deficits in cognitive ToM emerge prior to deficits in affective ToM, ${ }^{13}$ whereas others argue the opposite. ${ }^{8}$ The timing of these deficits may be connected with the type of skill captured within the task. For example, visuospatial abilities, which are typically utilized in affective ToM tasks, are also seen to decline early on in the disease process. ${ }^{1}$ Specific ToM tasks used to assess functioning may contribute to the inconsistent findings in this area as different subcomponents of ToM are assessed by different tests. Some ToM tasks rely on cognitive processing alone (ie, tasks that involve vignettes and pragmatic stories), whereas others rely on visual processing (ie, facial recognition, emotion recognition). The extent to which these tasks draw upon cognitive processing will determine the strength of the relationship between ToM deficits and executive functioning/cognitive decline.

A number of studies have sought to examine the association between executive function and ToM. However, as mentioned above, many tests of executive function and ToM processing also involve visuospatial components. The visuospatial deficits seen in PD patients have been associated with lesions in the right posterior cortex. ${ }^{21}$ Since visuospatial functioning deficits are evident early in the process of PD, it is plausible that visuospatial dysfunction may explain ToM deficits. ${ }^{22}$ Our study only included participants with adequate or corrected vision, therefore any deficits exhibited on the ToM task would not be due to pure visual deficits. The role of visuospatial deficits in recognizing emotional expression relates to how the images of the eyes are perceived as a whole. The test in this study involves processing the visual information in the pictures in an integrated manner-a process which may be greatly affected by visuospatial deficits.

Many have refuted the role of cognitive decline in ToM processing in $\mathrm{PD}$, with studies reporting deficits in ToM tasks (which relied heavily on visuospatial abilities) to be unrelated to executive functioning. ${ }^{10,12,14}$ Our study demonstrates that visuospatial functioning mediates the relationship between cognitive decline and ToM and is consistent with research on normal aging, which indicates that declines in social 
cognition and ToM processing is at least partially independent of more general cognitive or executive decline. ${ }^{23}$ It has been suggested that while it is not uncommon to see cognitive decline as a general consequence of normal aging, deficits in social cognition and ToM do not often follow. ${ }^{23,24}$ Considering that declines in ToM processing are not commonly associated with general cognitive decline in normal aging, it may be that these phenomena seen in PD patients are due to the additional deficits of visuospatial functioning. Furthermore, even when social cognitive processing has been reported in normal aging, it is not always related to general executive dysfunction. ${ }^{23}$

A point to consider regarding our findings is whether ToM deficits seen in PD are actually a display of the combined effects of visuospatial and cognitive deficits, both common in PD, and not ToM deficits at all. Indeed this is plausible considering ToM tests will either assess some form of cognitive processing or visuospatial processing, or both (as discussed above). However, this study found that cognitive functioning was not a significant predictor of ToM deficits when visuospatial abilities were considered in the analyses, which therefore suggests ToM is not purely a cognitive deficit. In effect, this implies that there are more to these deficits seen in PD patients than what would be seen from deficits in visuospatial functioning and cognition alone.

It is important to note the methodological weaknesses of our study. For example, the patients were only assessed during the medicated state, but research has shown that the medications can have a positive effect on cognitive function. Further, only one assessment of visuospatial functioning was utilized, which may restrict the ability to generalize results. The use of more than one visuospatial test may have provided more robust findings for the role of visuospatial processing in ToM. Similarly, in this study, only affective ToM was assessed. The test involved inferring one's emotions from a picture, which typically derives an index of affective as opposed to cognitive ToM processing. As a consequence, only affective ToM was assessed. Using a cognitive ToM task as well as the affective task would have enabled us to examine whether visuospatial deficits contribute to ToM difficulties as a whole. Further, the significant difference in sex ratio and depression symptomatology between the two participant groups was also of concern; however, these variables were controlled for as covariates and therefore not deemed to present any impact on the results of the study.

Future work in this area therefore should seek to replicate the results found in our study, as to date, no study has investigated the mediating role of visuospatial functioning in ToM in PD patients. More specifically, studies should include the use of other/multiple visuospatial assessment, beyond the line orientation task, to ensure these findings can be generalized. In addition, future studies should assess the mediating role of visuospatial functioning on both affective and cognitive ToM, and ToM as a whole in PD patients, through the use of multiple ToM tasks which utilize visual processing skills.

It may be concluded that visuospatial functioning plays an important mediating role for the relationship between executive dysfunction and affective ToM deficits in PD patients. Moreover, these results suggest that visuospatial deficits may directly contribute to the presence of ToM difficulties seen in individuals with PD, even in the early stages.

\section{Acknowledgments}

The research was supported by a grant from the Canterbury Medical Research Foundation.

\section{Disclosure}

The authors report no conflicts of interest in this work.

\section{References}

1. Poletti M, Enrici I, Bonuccelli U, Adenzato M. Theory of Mind in Parkinson's disease. Behav Brain Res. 2011;219(2):342-350.

2. McKinlay A, Grace RC, Dalrymple-Alford JC, Roger D. Characteristics of executive function impairment in Parkinson's disease patients without dementia. J Int Neuropsychol Soc. 2010;16(2):268-277.

3. Roca M, Manes F, Chade A, et al. The relationship between executive functions and fluid intelligence in Parkinson's disease. Psychol Med. 2012;42(11):2445-2452.

4. Mengelberg A, Siegert RJ. Is theory-of-mind impaired in Parkinson's disease? Cogn Neuropsychiatry. 2003;8(3):191-209.

5. Saltzman J, Strauss E, Hunter M, Archibald S. Theory of mind and executive functions in normal human aging and Parkinson's disease. J Int Neuropsychol Soc. 2000;6(7):781-788.

6. Eddy CM, Beck SR, Mitchell IJ, Praamstra P, Pall HS. Theory of mind deficits in Parkinson's disease: a product of executive dysfunction? Neuropsychology. 2013;27(1):37-47.

7. Yu RL, Wu RM, Chiu MJ, Tai CH, Li CH, Hua MS. Advanced theory of mind in patients at early stage of Parkinson's disease. Parkinsonism Relat Disord. 2012;18(1):21-24.

8. Santangelo G, Vitale C, Trojano L, et al. Neuropsychological correlates of theory of mind in patients with early Parkinson's disease. Mov Disord. 2011;27(1):98-105.

9. Kawamura M, Koyama S. Social cognitive impairment in Parkinson's disease. J Neurol. 2007;254(Suppl 4):IV/49-IV/53.

10. Tsuruya N, Kobayakawa M, Kawamura M. Is "reading mind in the eyes" impaired in Parkinson's disease? Parkinsonism Relat Disord. 2011;17(4):246-248.

11. Monetta L, Grindrod CM, Pell MD. Irony comprehension and theory of mind deficits in patients with Parkinson's disease. Cortex. 2009;45(8):972-981.

12. Bodden ME, Mollenhauer B, Trenkwalder C, et al. Affective and cognitive theory of mind in patients with Parkinson's disease. Parkinsonism Relat Disord. 2010;16(7):466-470.

13. Péron J, Vicente S, Leray E, et al. Are dopaminergic pathways involved in theory of mind? A study in Parkinson's disease. Neuropsychologia. 2009;47(2):406-414. 
14. Mimura M, Oeda R, Kawamura M. Impaired decision-making in Parkinson's disease. Parkinsonism Relat Disord. 2006;12(3):169-175.

15. Hoehn MM, Yahr MD. Parkinsionism: onset, progression and mortality. 1967. Neurology. 2001;57(10 Suppl 3):S11-S26.

16. Teng EL, Chui HC. The modified mini mental state (3MS) examination. J Clin Psychiatry. 1987;48(8):314-318.

17. Baron-Cohen S, Wheelwright S, Hill J, Raste Y, Plumb I. The "Reading the Mind in the Eyes" Test revised version: a study with normal adults, and adults with Asperger syndrome or high-functioning autism. JChild Psychol Psychiatry. 2001;42(2):241-251.

18. Benton AL, Varney NR, Hamsher KD. Visuospatial judgment: a clinical test. Arch Neurol. 1978;35(6):364-367.

19. Beck AT, Steer RA, Brown GK. Beck Depression Inventory, 2nd ed. San Antonio: Psychological Corporation; 1996.

20. Hayes AF. Introduction to Mediation, Moderation, and Conditional Process Analysis: A Regression-Based Approach. New York: Guilford Press; 2013.
21. Treccani B, Torri T, Cubelli R. Is judgement of line orientation selectively impaired in right brain damaged patients? Neuropsychologia. 2005;43(4):598-608.

22. Maeshima S, Itakura T, Nakagawa M, Nakai K, Komai N. Visuospatial impairment and activities of daily living in patients with Parkinson's disease: a quantitative assessment of the cube-copying task. Am J Phys Med Rehabil. 1997;76(5):383-388.

23. MacPherson SE, Phillips LH, Della Sala S. Age, executive function, and social decision making: A dorsolateral prefrontal theory of cognitive aging. Psychol Aging. 2002;17(4):598-609.

24. Maylor EA, Moulson JM, Muncer AM, Taylor LA. Does performance on theory of mind tasks decline in old age? Br J Psychol. 2002;93(Pt 4): 465-485.

\section{Publish your work in this journal}

Neuropsychiatric Disease and Treatment is an international, peerreviewed journal of clinical therapeutics and pharmacology focusing on concise rapid reporting of clinical or pre-clinical studies on a range of neuropsychiatric and neurological disorders. This journal is indexed on PubMed Central, the 'PsycINFO' database and CAS.
The manuscript management system is completely online and includes a very quick and fair peer-review system, which is all easy to use. Visit http://www.dovepress.com/testimonials.php to read real quotes from published authors. 